\title{
Primary hyperparathyroidism and Zollinger Ellison syndrome during pregnancy: a case report
}

\author{
Dalal Ali@1, Patrick Divilly1', Ruth Prichard1', Dermot O'Toole1,2,3, Donal O'Shea1,4 and \\ Rachel K Crowley 1,4
}

1St. Vincent's University Hospital, Dublin, Ireland, 2St. James's University Hospital, Dublin, Ireland, 3Trinity College, Dublin, Ireland, and 4 University College Dublin, Dublin, Ireland
Correspondence should be addressed to R K Crowley

Email

R.Crowley@st-vincents.ie

\begin{abstract}
Summary
Multiple endocrine neoplasia type 1 (MEN1) is a rare inherited endocrine disorder with a high rate of penetrance. The incidence of MEN1 is 1/30,000 in the general population; however, it is quite rare for a patient to present for medical attention with MEN1 for the first time in pregnancy. Primary hyperparathyroidism (PHPT) is one of the most common features of MEN1. The incidence of PHPT occurring in pregnancy is 1\%. Despite advances in the medical, surgical and obstetric care over the years, management of this condition during pregnancy may be challenging. It can be difficult to identify pregnant women with PHPT requiring intervention and to monitor safely. Hypercalcemia can result in significant maternal and fetal adverse outcomes including: miscarriage, intrauterine growth restriction, preterm delivery, neonatal hypocalcaemia, pre-eclampsia and maternal nephrolithiasis. Herein, we present a case study of a lady with a strong family history of MEN1, who was biochemically proven to have PHPT and evidence of Zollinger Ellison Syndrome (ZE) on endoscopy. This patient delayed her assisted pregnancy plans for in vitro fertilization (IVF) until completion of the MEN1 workup; nevertheless, she spontaneously achieved an unplanned pregnancy. As a result, she required intervention with parathyroidectomy in the second trimester of her pregnancy as her calcium level continued to rise. This case study highlights the workup, follow up and management of MEN1 presenting with PHPT and ZE in pregnancy.
\end{abstract}

\section{Learning points:}

- Women of childbearing age who are suspected to have a diagnosis of primary hyperparathyroidism ideally should have genetic testing and avoid pregnancy until definitive plans are in place.

- Zollinger Ellison syndrome in pregnancy means off-label use of high dose of proton pump inhibitors (PPI). Use of PPI in pregnancy is considered to be safe based on retrospective studies. Omeprazole, however, is FDA class C drug because of lack of large prospective studies or large case series during pregnancy.

- Calcium supplements in the form of calcium carbonate must be converted to calcium chloride by gastric acid in order to be absorbed, however, patients rendered achlorhydric as a result of PPI use will have impaired absorption of calcium. Therefore, use of calcium citrate might be considered a better option in this case.

\section{Background}

MEN1 is a rare genetic endocrine disorder. It is inherited in an autosomal dominant fashion. There is no gender or ethnic predilection; however, a significant proportion of these cases present in women of childbearing age. This leads to rare and difficult clinical challenges in pregnant women. The incidence of sporadic PHPT 
occurring in pregnancy is reported as $1 \%(1,2)$. It is well reported that the most common form of PHPT will present as a solitary parathyroid adenoma and less than $2 \%$ will have 2 or more lesions (2). The latter is often seen in patients with an underlying genetic mutation. The greatest difficulty is to control maternal calcium level during pregnancy as calcimimetics have not been approved for use in pregnancy (3). It is reasonable to manage mild cases of hypercalcemia conservatively, but surgery may be indicated for moderate to severe cases. In non-pregnant patients, localization of the adenoma is performed by a combination of ultrasound with ionising radiation (99mTc Sestamibi scan (gold standard)/CT scan). Ultrasound is the only radiological modality that can be used in pregnancy to localize the site/sites of the disease. This is a unique case of MEN1 manifested as PHPT, with hypoparathyroidism post-operatively, and ZE syndrome in pregnancy all of which were challenging and were managed with a multidisciplinary (MDT) approach.

\section{Case presentation}

\section{Medical history and demographics}

We report the case of a 34-year-old lwoman who was referred to our service from a fertility clinic. As part of her workup for primary infertility, it was noted that her adjusted calcium was elevated at $2.84 \mathrm{mmol} / \mathrm{L}$ (normal range: $2.20-2.60 \mathrm{mmol} / \mathrm{L}$ ) and $\mathrm{PTH}$ was elevated at $84.4 \mathrm{ng} / \mathrm{L}$ (normal range: 15-65 ng/L) (Fig. 1). She had no significant past medical history and was not on regular medications. Of note, she had no renal or bone disease. Her family history was strongly positive for MEN 1 with a genetically proven case in her brother and her mother and five out of six maternal aunts and uncles with clinical diagnosis of MEN 1. The patient was evaluated at a neuroendocrine tumour (NET) clinic and was investigated for MEN 1, with goal to correct any endocrine abnormalities prior to undergoing further treatment for fertility.

\section{Investigation}

As part of the workup, it was noted that Gastrin level was elevated at $208 \mathrm{pg} / \mathrm{mL}$ (normal range: 29.4-121 pg/ $\mathrm{mL}$ ) and chromogranin A was $376 \mathrm{mg} / \mathrm{mL}$ (45-150 mg/ $\mathrm{mL})$. IGF-1, Insulin and glucagon were all within the normal range. The patient underwent endoscopy and endoscopic ultrasound (EUS); features were consistent with ZE syndrome; two to three $4-5 \mathrm{~mm}$ hypoechoic lesions consistent with small pNETs in the body and tail of the pancreas, and multiple shallow ulcers in the duodenum (Fig. 2). No obvious tumour in the head of the pancreas was identified and there were no suspicious nodes. She had a CT abdomen which showed a $1.2 \mathrm{~cm}$ nodule in the left adrenal gland. Adrenal endocrine functional workup was normal. Additionally, she had an octreotide scan which showed no site of active disease. She had a normal DXA scan and MRI of the pituitary. 99mTc Sestamibi scan demonstrated no focal tracer uptake in superior mediastinum or neck, ultrasound neck demonstrated a hypervascular ovoid nodule measuring $1.5 \mathrm{~cm}$ at lower pole right lobe of thyroid suspicious for a parathyroid adenoma (Fig. 3) and so the patient was referred to the endocrine surgeons for further management.

Unfortunately, when the patient presented to the surgical clinic; 12 weeks following previous investigations, she was found to be 7 weeks' pregnant. Her calcium levels were monitored closely throughout her pregnancy and her ionised calcium remained elevated at $1.52 \mathrm{nmol} / \mathrm{L}$ (normal range: $1.19-1.35 \mathrm{nmol} / \mathrm{L}$ ) with

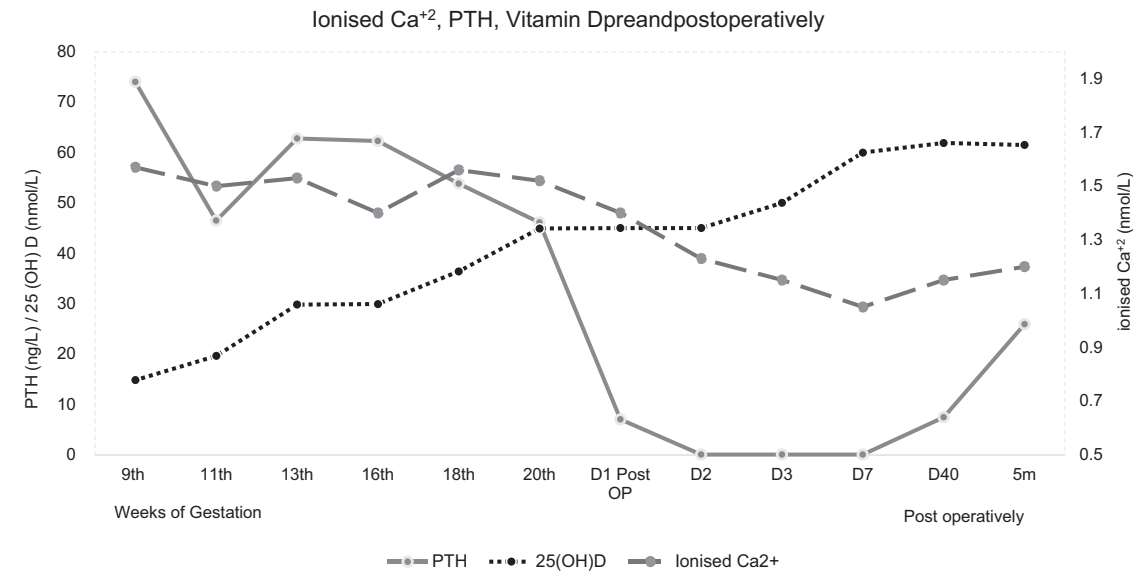

Figure 1

Pre- and post-operative ionised $\mathrm{Ca}^{+2}$, PTH, Vitamin $\mathrm{D}$ and phosphate. 


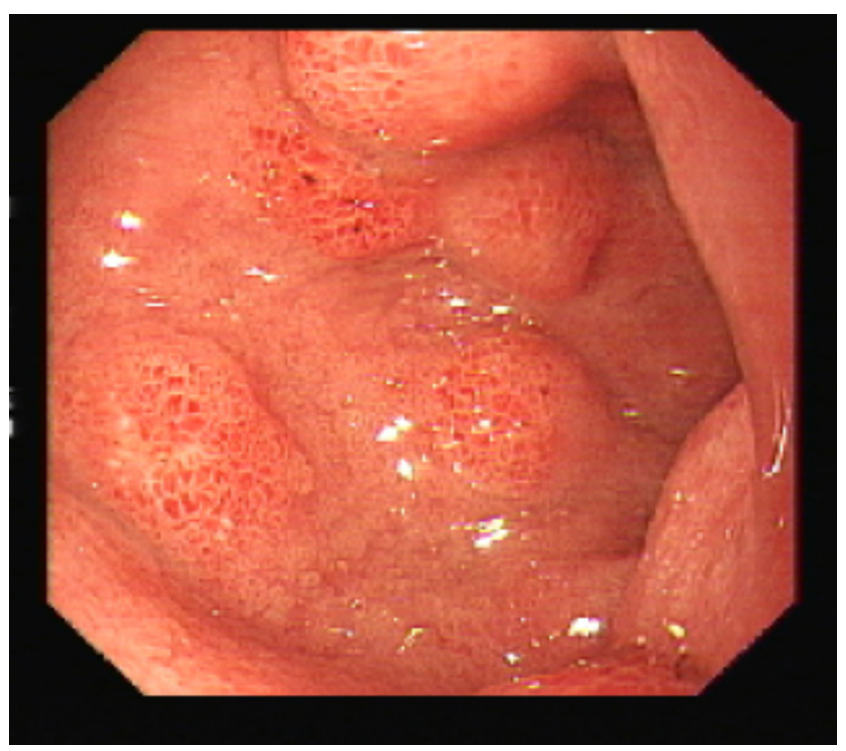

Figure 2

Endoscopic ultrasound image revealing multiple shallow lesions in the duodenum.

elevated parathyroid hormone (PTH) at $74 \mathrm{ng} / \mathrm{L}$ (normal range: $15-65 \mathrm{ng} / \mathrm{L}$ ) and low vitamin $\mathrm{D}$ of $14.8 \mathrm{nmol} / \mathrm{L}$ (desired greater than $50 \mathrm{nmol} / \mathrm{L}$ ). Given the concerns with regards to the use of cinacalcet in pregnancy, the patient was monitored with weekly calcium and PTH levels. She was also closely monitored for hypertension and any signs of foetal growth restriction throughout the pregnancy. Her genetic screening confirmed c831del mutation in MEN gene.

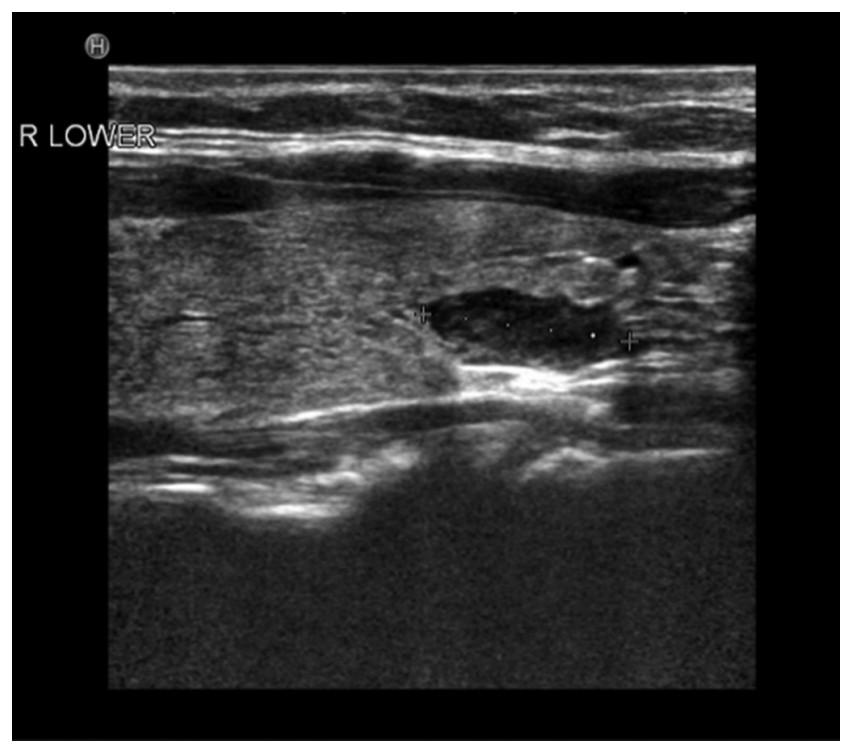

Figure 3

Ultrasound neck showinghypervascular ovoid nodule.

\section{Treatment}

The patient was initially commenced on high dose PPI (omeprazole $40 \mathrm{mg}$ twice daily) following her endoscopic findings. The NET MDT was contacted by the maternal foetal medicine team at 24 weeks' gestation with concerns re the continued rise in calcium levels. After reviewing the patient with an MDT approach, she was admitted electively for parathyroid exploration and subtotal parathyroidectomy. Fluid rehydration alone failed to suppress her calcium level. The procedure went well and three glands were removed, leaving the right upper gland in situ.

\section{Outcome and follow-up}

Post-operatively, the patient was transferred to ICU and commenced on IV calcium gluconate infusion. At this time, she was also commenced on oral calcium carbonate and alfacalciferol. The patient was transferred to the wards, with mother and fetus doing well. Despite increasing doses of calcium carbonate, ionised calcium levels were not maintained off infusion with the patient frequently becoming symptomatic with peri-oral paraesthesia and positive Chvostek's sign. Magnesium replacement was also commenced. The patient remained dependent on IV calcium infusion for the first 12 postoperative days, fetus viability was closely monitored during this time. It was suggested that the patient was not absorbing calcium carbonate due to PPI use, therefore, she was switched to calcium citrate, a non-standard form of oral calcium in Ireland. IV calcium replacement was ceased 2 days later and the patient delivered at 37 weeks a healthy baby with no adverse events. Parathyroid axis recovered and the patient did not require calcium/vitamin D supplements for maintenance. She has since had a second uncomplicated pregnancy.

\section{Discussion}

Diagnosis of PHPT in pregnancy is rare, as is MEN1. There are only 155 cases reported in the literature of PHPT diagnosed in pregnancy (5). The majority of cases of PHPT in pregnancy can be managed with surveillance. It is important to establish a threshold for intervention with maternal foetal medicine. Untreated moderate to severe hypercalcemia can cause potential adverse outcomes including foetal and maternal deaths, preeclampsia and pregnancy-induced hypertension $(6,7)$. In our patient, the need for surgical intervention was due 
to failure to optimise calcium levels with conservative measures. Genetic screening informed the decision for three gland-parathyroidectomy. As our patient remained dependent on IV calcium for a few days post-operatively and failed to switch to oral calcium successfully, we changed the calcium salt to achieve successful result. For the absorption of calcium carbonate to happen, calcium carbonate must be converted to calcium chloride by gastric acid. In patients rendered achlorhydric, as with our patient secondary to high dose PPI, gastric acid levels are greatly reduced meaning that little calcium carbonate is converted into its absorbable form. In these instances, the bioavailability of the calcium carbonate drops from $30 \%$ to approximately $4 \%$. Calcium citrate does not require gastric acid to be absorbed and this maintains its absorption rates in this patient population. In fact, calcium carbonate is the standard form of oral calcium replacement in Ireland, hence, it was one of the challenges we faced when switching the patient to calcium citrate while pregnant. Another challenge was the use of high dose PPI and its safety profile in pregnancy. This was thoroughly reviewed in the literature and PPI remain classified as class $\mathrm{B} / \mathrm{C}$ in pregnancy due to lack of prospective studies but the overall profile is described as safe (8).

\section{Declaration of interest}

The authors declare that there is no conflict of interest that could be perceived as prejudicing the impartiality of this case report.

\section{Funding}

This research did not receive any specific grant from any funding agency in the public, commercial or not-for-profit sector.

\section{Patient consent}

A written informed consent has been obtained from the patient for publication of the submitted article and accompanying images.

\section{Author contribution statement}

$D A$ and $P D$ wrote the first draft of this manuscript and were involved in collection of clinical data. All authors were involved in the writing of this manuscript and final review of the manuscript. R K C, R P, D O and D O were involved in the clinical care of these patients and collection of data.

\section{References}

1 McCarthy A, Howarth S, Khoo S, Hale J, Oddy S, Halsall D, Fish B, Mariathasan S, Andrews K, Oyibo SO, et al. Management of primary hyperparathyroidism in pregnancy: a case series. Endocrinology, Diabetes and Metabolism Case Reports 20192019 190039. (https://doi. org/10.1530/EDM-19-0039)

2 Gokkaya N, Gungor A, Bilen A, Bilen H, Gviniashvili D \& Karadeniz Y. Primary hyperparathyroidism in pregnancy: a case series and literature review. Gynecological Endocrinology 201632 783-786. (https://doi.org/10.1080/09513590.2016.1188916)

3 Amaya García M, Acosta Feria M, Soto Moreno A, Dios Fuentes E, Navarro González E, Quijada Thong D, Del Valle A, Acosta Delgado D \& Astorga Jiménez R. Primary hyperparathyroidism in pregnancy. Gynecological Endocrinology 200419 111-114. (https://doi. org/10.1080/09513590400002334)

4 Djokanovic N, Klieger-Grossmann C \& Koren G. Does treatment with bisphosphonates endanger the human pregnancy? Journal of Obstetrics and Gynaecology Canada 200830 1146-1148. (https://doi. org/10.1016/S1701-2163(16)34026-9)

5 DiMarco A, Meeran K, Christakis I, Sodhi V, Nelson-Piercy C, Samuel Tolley N \& Palazzo FF. Seventeen Cases of Primary Hyperparathyroidism in Pregnancy: A Call for Management Guidelines. Journal of the Endocrine Society 20193 1009-1021. (https://doi.org/10.1210/js.2018-00340)

6 Fouda MA. Primary hyperparathyroidism and pregnancy. Saudi Medical Journal 200021 31-35.

7 Schnatz PF \& Curry SL. Primary hyperparathyroidism in pregnancy: evidence-based management. Obstetrical and Gynecological Survey 2002 57 365-376. (https://doi.org/10.1097/00006254-200206000-00022)

8 Thélin CS \& Richter JE. Review article: the management of heartburn during pregnancy and lactation. Alimentary Pharmacology and Therapeutics 202051 421-434. (https://doi.org/10.1111/apt.15611)

Received in final form 2 August 2020

Accepted 25 January 2021 